\title{
Acute kidney injury caused by ammonium acid urate crystals in diabetic ketoacidosis at the onset of type 1 diabetes mellitus
}

\author{
Shunsuke Shimazaki, Itsuro Kazukawa, Kyoko Mori, Makiko Kihara and \\ Masanori Minagawa
}

Department of Endocrinology, Chiba Children's Hospital, Chiba, Japan
Correspondence should be addressed to I Kazukawa

Email

enddmcch@gmail.com

\section{Summary}

Ammonium acid urate (AAU) crystals are rare in industrialized countries. Furthermore, the number of children with diabetic ketoacidosis (DKA) who develop severe acute kidney injury (AKI) after hospitalization is small. We encountered two patients with AKI caused by AAU crystals during the recovery phase of DKA upon admission. They were diagnosed with severe DKA and hyperuricemia. Their urine volume decreased and AKI developed several days after hospitalization; however, acidosis improved in both patients. Urine sediment analysis revealed AAU crystals. They were treated with urine alkalization and diuretics. Excretion of ammonia in the urine and urine $\mathrm{pH}$ levels increased after treatment of DKA, which resulted in the formation of AAU crystals. In patients with severe DKA, the urine and urine sediment should be carefully examined as AAU can form in the recovery phase of DKA.

\section{Learning points:}

- Ammonium acid urate crystals could be formed in the recovery phase of diabetic ketoacidosis.

- Diabetic ketoacidosis patients may develop acute kidney injury caused by ammonium acid urate crystals.

- Urine and urine sediment should be carefully checked in patients with severe DKA who present with hyperuricemia and volume depletion.

\section{Background}

Ammonium acid urate (AAU) crystals are rare in industrialized countries $(1,2)$. Several studies have reported that the risk factors of AAU crystals include laxative abuse, urinary tract infection, and morbid obesity $(1,3)$.

A previous study reported acute kidney injury (AKI) in children with diabetic ketoacidosis (DKA), in whom AKI was caused by urine volume depletion and severe acidosis (4); a majority of them recovered with volume and insulin infusions and did not develop severe AKI after hospitalization. We previously reported a patient with AKI caused by AAU crystals (5). AKI caused by AAU crystals with increased severity during the recovery phase of DKA is rare. Here, we report a series of two DKA patients with AKI caused by AAU crystals.

\section{Case presentation}

\section{Case 1}

Case 1 was a 14-year-old boy who had gained weight due to lack of exercise and overeating. His history revealed polyuria, polydipsia, and a documented $13 \mathrm{~kg}$ weight loss in the month preceding admission. He presented at the emergency unit of our hospital with confusion and difficulty in walking. He was tachypneic (40 breaths 
per minute) with Kussmaul breathing, his heart rate was 140 b.p.m., blood pressure was $77 / 44 \mathrm{mmHg}$, and body temperature was $36.7^{\circ} \mathrm{C}$. He was moderately obese, weighing $74.0 \mathrm{~kg}$ (excess weight $+21.1 \%$ ) and was 174.0 $\mathrm{cm}(+1.58$ s.D. $)$ tall.

\section{Case 2}

Case 2 was a 4-year-old girl. She had polyuria for the month preceding admission. She was transferred to the emergency unit of our hospital by ambulance and presented with confusion. She was tachypneic (40 breaths per minute) with Kussmaul breathing and a heart rate of 148 b.p.m., blood pressure of 122/78 mmHg, and temperature of $36.5^{\circ} \mathrm{C}$. She was of medium build, weighing $18 \mathrm{~kg}$ (excess weight $-7.7 \%$ ), and was $112.0 \mathrm{~cm}$ $(+1.83$ s.D. $)$ tall.

\section{Investigation}

\section{Case 1}

Severe DKA was confirmed (6) (Table 1) and we found uraturia in his urine sediment. He was diagnosed with stage 2 AKI due to the serum level of creatinine (7). Immediate treatment consisted of insulin infusion, intravenous saline, and extracellular fluid. He was awake the following day. However, 3 days after initiation of therapy, his urine output decreased to $0.04 \mathrm{~mL} / \mathrm{kg} / \mathrm{h}$, and he developed stage 3 AKI. His venous $\mathrm{pH}$ was 7.476, serum creatinine level was $2.7 \mathrm{mg} / \mathrm{dL}$, and urine analysis revealed a $\mathrm{pH}$ of 6.5. Analysis of urine crystals at this time revealed AAU. The fractional excretion of uric acid (FEUA) was low (4\%), and his fractional excretion of sodium (FENa) was $0.71 \%$.
Abdominal plain CT revealed high-density areas in the bilateral renal medulla and left ureteral stones. We found no hydronephrosis or hydroureter.

\section{Case 2}

Severe DKA was diagnosed, but AKI was not confirmed (Table 1); initial treatment consisted of insulin infusion, intravenous saline, and extracellular fluid administration. Her urine volume decreased, and she developed stage 2 AKI (serum creatinine level was $0.49 \mathrm{mg} / \mathrm{dL}$ and urine output was $0.35 \mathrm{~mL} / \mathrm{kg} / \mathrm{h}$ ) $30 \mathrm{~h}$ after initiation of therapy when her venous $\mathrm{pH}$ was 7.393. Eighteen hours after admission, urine analysis showed that her $\mathrm{pH}$ was 6.0, FEUA increased (14.7\%), and FENa was 0.6\%. However, at the onset of AKI, her $\mathrm{pH}$ was 6.5, FEUA was remarkedly decreased $(2.3 \%)$, and FENa was very low $(0.18 \%)$. We also found reddish-brown urine sediments on insertion of the urethral catheter (Fig. 1). Analysis of urine crystals revealed AAU. We found no stones in the renal medulla or ureters on abdominal plain CT.

\section{Treatment}

\section{Case 1}

He was diagnosed with AKI due to kidney crystals, which were treated with urine alkalization and diuretics.

\section{Case 2}

She was treated with urine alkalization and diuretics.

Table 1 Main laboratory results obtained on admission of patients to our hospital.

Blood glucose level ( $\mathrm{mmol} / \mathrm{L})$

Hemoglobin $\mathrm{A}_{1 \mathrm{c}}$ (\%)

Blood beta-hydroxybutyrate $(\mathrm{mmol} / \mathrm{L})$

$\mathrm{pH}$

$\mathrm{pCO}_{2}(\mathrm{kPa})$

Bicarbonate ( $\mathrm{mmol} / \mathrm{L})$

Sodium (mmol/L)

Chloride $(\mathrm{mmol} / \mathrm{L})$

Potassium (mmol/L)

BUN (mg/dL)

Creatinine $(\mathrm{mg} / \mathrm{dL})$

$\mathrm{UA}(\mathrm{mg} / \mathrm{dL})$

Serum insulin $(\mu \mathrm{U} / \mathrm{mL})$

Anti-glutamic acid decarboxylase antibody $(\mathrm{U} / \mathrm{mL})$

Anti-IA-2 antibody (U/mL)

\begin{tabular}{cccc}
\hline Case $\mathbf{1}$ & & Case $\mathbf{2}$ \\
\cline { 1 - 1 } 40.6 & & 31.0 \\
11.8 & & 13.2 \\
2.3 & & 5.6 \\
7.02 & & 6.88 \\
3.61 & & 17.2 \\
6.9 & & 3.2 \\
145 & & 136 \\
104 & & 101 \\
4.2 & & 4.4 \\
35.0 & & 14.5 \\
0.95 & & 0.35 \\
19.2 & & 9.5 \\
10.6 & & 0.93 \\
9.0 & & 6.9 \\
$<0.4$ & & $>30$ \\
& &
\end{tabular}

\begin{tabular}{c}
\hline Reference value \\
\hline $3.9-7.0$ \\
$<7$ \\
$\geq 0.2$ \\
$7.35-7.45$ \\
$4.66-6.0$ \\
$21-26$ \\
$135-145$ \\
$98-106$ \\
$3.5-5.9$ \\
$6-20$ \\
$0.4-1.1$ \\
$2.3-7.5$ \\
\\
$\geq 5.0$ \\
$\geq 0.4$
\end{tabular}

BUN, blood urea nitrogen; IA-2, insulinoma-associated antigen-2; UA, uric acid 


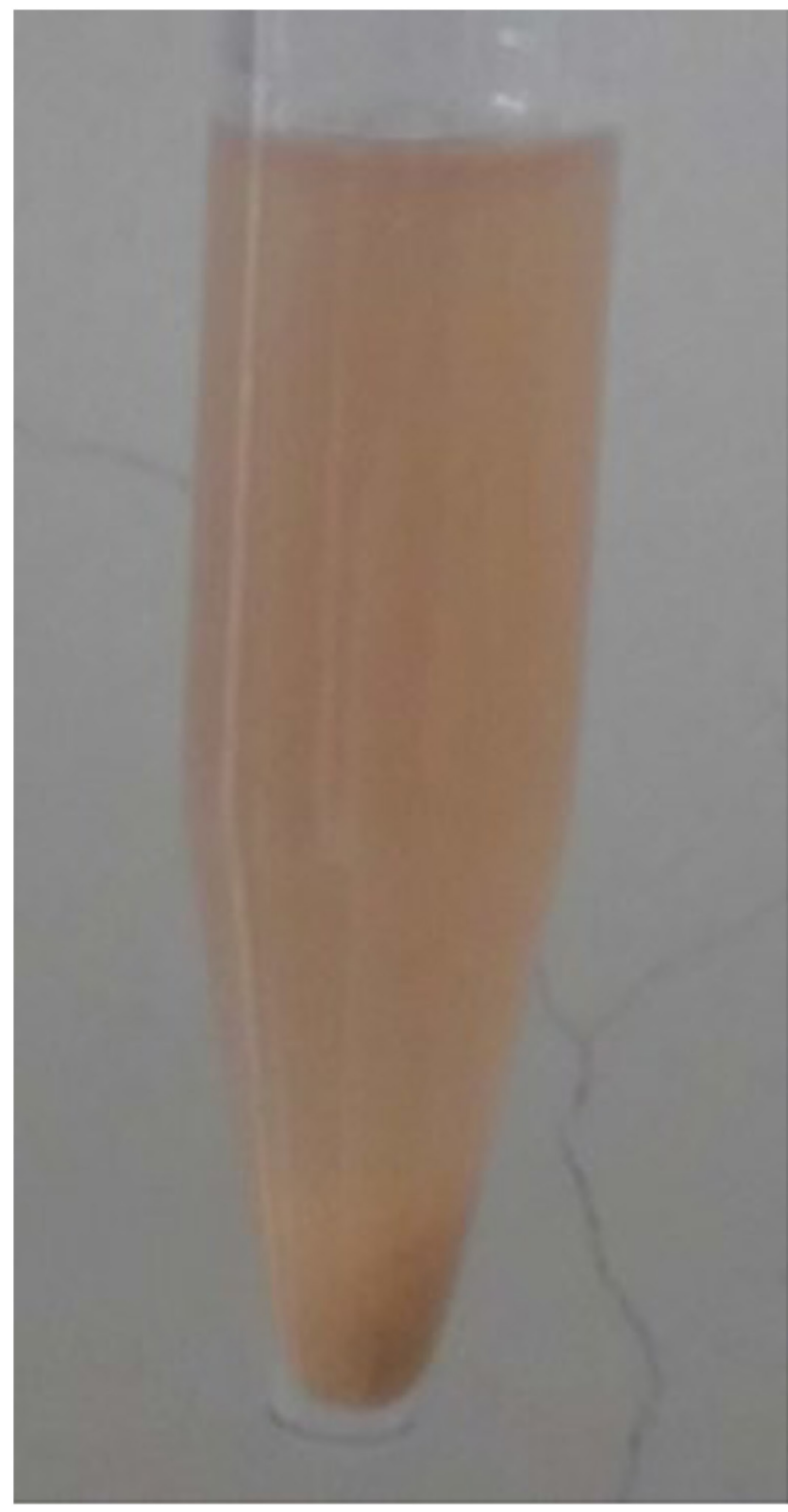

Figure 1

Ammonium acid urate crystals in the urine of Case 2. Reddish-brown segments are seen in the urine on insertion of the urethral catheter.

\section{Outcome and follow-up}

\section{Case 1}

His urine volume increased on hospital day 4. FEUA increased (14\%), and the UA serum was decreased (2.6 $\mathrm{mg} / \mathrm{dL}$ ) on hospital day 8 . He was diagnosed with type 1 diabetes mellitus (DM) by the antibody test (Table 1).

\section{Case 2}

Twenty-four hours after these treatments, her urine volume, FEUA (5.9\%), and FENa (1.41\%) increased, and the serum level of UA decreased $(2.2 \mathrm{mg} / \mathrm{dL})$. She was diagnosed with type $1 \mathrm{DM}$ by the antibody test (Table 1 ).

\section{Discussion}

The proportion of children hospitalized for DKA who develop AKI is $64.2 \%$ (4). However, few patients develop increased AKI severity between 24 and $48 \mathrm{~h}$ after hospitalization. Our patients developed AKI severity after admission, even though their acidosis improved.

The serum levels of UA in our patients were high upon admission (Table 1). In DKA, hyperketonemia accelerates the uptake of uric acid by urate transporter 1 and reabsorption of uric acid by glucose transporter 9 (8). Additionally, the expression of organic anion transporter 1 and 3 is decreased in hyperuricemia. Further, sodium-dependent phosphate cotransporter 1 and 4 activity is decreased because the transporters are $\mathrm{Cl}$-dependent, and the ketone bodies suppress the vesicular glutamate transporter activity (9). Hence, DKA is indicative of hyperuricemia.

The urinary excretion of ammonia reaches a maximum only 5-6 days after the onset of acidosis (1). Further, a previous study has shown that AAU crystals disappear from urine when the $\mathrm{pH}$ is lower than 5.7 and that the minimum concentration of AAU crystals formed at $\mathrm{pH}$ levels between 6.2 and 6.3 (3). In our patients, it is likely that the urinary excretion of ammonium reached a maximum value when the AAU crystals were found; however, we were unable to check the ammonium level in the urine.

The blood volume depletion induces a decrease in urine volume and urine sodium. The decrease in urine sodium in patients with laxative abuse induces coupling of AAU crystals by excretion of urinary ammonia (3). We observed low urinary sodium in Case 2 at the onset of AKI; similar mechanisms for the formation of AAU crystals might be found in patients with DKA.

We found that AAU crystals could be formed in the recovery phase of DKA. In conclusion, we suggest that physicians should carefully check urine and urine sediment in patients with severe DKA who present with hyperuricemia and volume depletion, even if their acidosis is improved.

\section{Declaration of interest}

The authors declare that there is no conflict of interest that could be perceived as prejudicing the impartiality of the research reported.

\section{Funding}

This research did not receive any specific grant from any funding agency in the public, commercial or not-for-profit sector. 


\section{Patient consent}

Written informed consent was obtained from the patient for publication of this case report.

\section{Author contribution statement}

Shunsuke Shimazaki, Itsuro Kazukawa, Kyoko Mori, Makiko Kihara, and Masanori Minagawa were involved in the patient care and drafted the case report.

\section{References}

1 Shirasu A, Ashida A, Matsumura H, Nakakura H \& Tamai H. Clinical characteristics of rotavirus gastroenteritis with urinary crystals. Pediatrics International 201557 917-921. (https://doi.org/10.1111/ped.12626)

2 Lomas DJ, Jaeger CD \& Krambeck AE. Profile of the ammonium acid urate stone former based on a large contemporary cohort. Urology 2017102 43-47. (https://doi.org/10.1016/j.urology.2016.10.027)

3 Dick WH, Lingeman JE, Preminger GM, Smith LH, Wilson DM \& Shirrell WL. Laxative abuse as a cause for ammonium urate renal calculi. Journal of Urology 1990143 244-247. (https://doi. org/10.1016/s0022-5347(17)39923-8)
4 Hursh BE, Ronsley R, Islam N, Mammen C \& Panagiotopoulos C. Acute kidney injury in children with type 1 diabetes hospitalized for diabetic ketoacidosis. JAMA Pediatrics 2017171 e170020. (https:// doi.org/10.1001/jamapediatrics.2017.0020)

5 Ishii T, Kazukawa I, Hisano M, Ishida M \& Minagawa M. A 14-yearold boy case of acute renal failure caused by ammonium acid urate crystals during the recovery phase of diabetic ketoacidosis at the time of type 1 diabetes mellitus onset. Japanese Journal of Emergency Pediatrics 201817 482-486 (in Japanese).

6 Wolfsdorf JI, Glaser N, M, Fritsch M, Hanas R, Rewers A, Sperling MA \& Codner E. ISPAD clinical practice consensus guidelines 2018: diabetic ketoacidosis and the hyperglycemic hyperosmolar state. Pediatric Diabetes 201827 (Supplement 27) 155-177.

7 Kidney Disease Improving Global Outcomes (KDIGO) Acute Kidney Injury Working Group. KDIGO clinical practice guideline for acute kidney injury. Kidney International 2012 (Supplement 2) 1-138. (https://doi.org/10.1038/kisup.2012.2)

$8 \mathrm{Xu}$ L, Shi Y, Zhuang S \& Liu N. Recent advances on uric acid transporters. Oncotarget 20178 100852-100862. (https://doi. org/10.18632/oncotarget.20135)

9 Iharada M, Miyaji T, Fujimoto T, Hiasa M, Anzai N, Omote H \& Moriyama Y. Type 1 sodium-dependent phosphate transporter (SLC17A1 protein) is a $\mathrm{Cl}^{-}$-dependent urate exporter. Journal of Biological Chemistry 2010285 26107-26113. (https://doi. org/10.1074/jbc.M110.122721)

Received in final form 22 January 2021

Accepted 2 February 2021 